Interventions/Outcome measures Demographic data, selfreported questionnaire and clinical oral examination were recorded and collected.

Result We recruited 249 athletes with the median age of 20 (range 13-43). Active caries (ICDAS code $\geq 3$ ) was found in $66.8 \%$ athletes with periodontal diseases (BPE code $\geq 1$ ) in $84.9 \%$. A quarter of those with both diseases were youth athletes. Pericoronitis was also found in $4.7 \%$ athletes while $9 \%$ have pulpitis, abscess or ulcer (PUFA). Approximately 23.2\% of athletes felt their oral health was not good during data collection with $32.5 \%$ reported moderate-to-severe impact of oral-related problems on their sports performance over the past 12 months: oral pain (10.5\%), difficulty participating in training and competition (11.4\%), performance affected $(6.4 \%)$ and reduction in training volume (6.3\%). Chi-square test of independence showed that there is a relationship between both diseases with ethnicity and sports type (individual-team or endurance-strength-mixed). Periodontal diseases were also seen related to age and education level. Approximately $5.5 \%$ of the athletes reported never seeing a dentist before.

Conclusion High levels of oral diseases were found in Malaysian elite athletes with common self-reported impacts on performance. This study hopes to create oral health awareness among Malaysian athletes and authorities so that it will be proportionately integrated into athlete health programmes in the future.

\section{THE INFLUENCE OF SUBCLINICAL HYPOTHYROIDISM ON PHYSICAL PERFORMANCE OF ELITE ATHLETES}

${ }^{1}$ Elena Tenyaeva, ${ }^{1,2}$ Elena Turova, ${ }^{1}$ Albina Golovach, ${ }^{1,2}$ Victoria Badtieva, ${ }^{1}$ Irirna Artikulova ${ }^{1}$ Moscow Scientific and Practical Center for Medical Rehabilitation, Restorative and Sports Medicine of the Moscow Department of Health, Moscow, Russian Federation; ${ }^{2}$ I.M. Sechenov First Moscow State Medical University, Moscow, Russian Federation

\subsection{6/bjsports-2021-IOC.415}

Background Currently, there is no consensus on the frequency of subclinical hypothyroidism in athletes and its effect on exercise tolerance.

Objective The purpose of the study was to explore the prevalence of subclinical hypothyroidism in elite athletes and to identify its impact on physical performance indicators.

Main Outcome Measurements A retrospective analysis of data from a random sample of outpatient records of 1000 elite athletes aged 15 to 36 years who underwent medical screening, including clinical, laboratory and instrumental examinations.

Results According to the results of a laboratory study, subclinical hypothyroidism was detected in 95 (9.5\%) athletes in the sample. In athletes with subclinical hypothyroidism, the average thyroid stimulating hormone (TSH) level was $5.53 \pm 0.24 \mathrm{mME} / \mathrm{l}$, while in unaffected athletes it was 1.89 $\pm 0.31 \mathrm{mME} / \mathrm{l}(\mathrm{p}<0.01)$. The level of free T4 was within normal values and in the group with hypothyroidism was $12.0 \pm 0.48 \mathrm{pM} / 1$, whereas in unaffected athletes $17.2 \pm 1.13$ $\mathrm{pM} / 1(\mathrm{p}<0.05)$.

When analyzing bicycle ergometry data, a significant correlation was found between hypothyroidism and heart rate at $1 \mathrm{st}, 3 \mathrm{rd}$ and 5 th minutes of recovery after the test $(\mathrm{p}<0.001)$, and with diastolic blood pressure at the 3rd minute of recovery $(\mathrm{p}<0.001)$. A significant positive correlation was also found between TSH level and the same set of recovery indicators $(\mathrm{p}<0,0001)$. There was also a significant negative relationship between the level of TSH and the intensity of the training regime $(\mathrm{p}<0.005)$ and with sports proficiency grade $(\mathrm{p}<0.0001)$.

We did not find any significant effect of TSH and hypothyroidism on exercise tolerance and aerobic reserve.

Conclusions The study showed a high prevalence of subclinical hypothyroidism in elite athletes, affecting 9.5\% of the sample. The presence of subclinical hypothyroidism significantly contributed to a slower recovery of parameters of the cardiovascular system after at bicycle ergometer test, without affecting exercise tolerance.

\section{THE EFFECT OF SLEEP ON THE PREVALENCE OF SPORTS INJURIES IN ATHLETES}

${ }^{1,2}$ Peter Vermeir, 'Loïs Arickx, ${ }^{1}$ Emely De Clercq, ${ }^{1}$ Anse De Landsheer, ${ }^{1}$ Ruben Vermeir,
1,2 Luc Vanden Bossche, ${ }^{1,2}$ An Mariman. ' Ghent University, Faculty of Medicine and
Healthcare sciences, Ghent, Belgium; ${ }^{2}$ Ghent University Hospital, Ghent, Belgium

10.1136/bjsports-2021-IOC.416

Background Many sports athletes are injured every year. Sleep quality and quantity play an important role in this.

Objectives A systematic review was carried out on the correlation between sleep and the prevalence of sports injuries.

Design Systematic review

Methods Screening of articles in PubMed, Web of Science, Cinahl and Cochrane Library on the keywords 'Sleep', 'Circadian rhythm', 'Insomnia', 'Jetlag', '(Elite) athletes', '(Sports) injuries' and 'Rehabilitation' published between January 1, 2010 and December 3, 2020. Systematic review made after assessment of the articles for methodological quality.

Results In general, athletes do not meet the total sleep time recommended by the American Academy of Sleep Medicine (AASM) and the National Sleep Foundation. One of the reasons for an increased risk of sports injuries is sleep deprivation. Sometimes sleep extension is needed to partially repair this by scheduling a short nap of about 30 minutes in the morning or early afternoon. In addition to sleep quantity, poor sleep quality also plays a role in the risk of sports injuries. Both, sleep quantity and quality, are negatively affected by air travel over different time zones, which is further enhanced by a heavy training schedule.

Conclusion Poor sleep quantity and/or quality have a negative effect on the prevalence of sports injuries. Sleep deprivation adversely affects sports-related parameters including physiological biomarkers related to injuries. In addition to sleep quality and quantity, training modalities, injury history, sleep disorders, gender, well-being and health are also associated with injury risks. Further research is needed to clarify the correlation between sleep and injury risk and to formulate practical recommendations.

\section{THE IMPACT OF SLEEP ON THE RECOVERY OF SPORT INJURIES}

${ }^{1,2}$ Peter Vermeir, ${ }^{1}$ Margot De Leye, ${ }^{1}$ Robbe Grymonprez, ${ }^{1}$ Arthur Goethals, ${ }^{1}$ Ruben Vermeir,

1,2 Luc Vanden Bossche, ${ }^{1,2} \mathrm{An}$ Mariman. 'Ghent University, Faculty of Medicine and Healthcare sciences, Ghent, Belgium; ${ }^{2}$ Ghent University Hospital, Ghent, Belgium

10.1136/bjsports-2021-IOC.417 